\title{
Cofilin-1 is involved in regulation of acting reorganization during influenza A virus assembly and budding
}

Ge Liu ${ }^{a}$, Yang-Fei Xiang ${ }^{b}$ Chao-Wan Guo ${ }^{a}$, Ying Pei ${ }^{b}$, Yi-Fei Wang ${ }^{b *}$, and Kaio Kitazato ${ }^{\text {* }}$

a. Division of Molecular Pharmacology of Infectious agents, Department of Molecular Microbiology and Immunology, Graduate School of Biomedical Sciences, Nagasaki University, 852-8521, Nagasaki, Japan.

b. Biomedical R\&D Center, Guangdong Provincial Key Laboratory of Bioengineering Medicine, National Engineering Research Center of Genetic Medicine, Jinan University, Guangzhou,510632, Guangdong, China.

*Corresponding author. Tel: +81 95819 2457; Fax: +81958192898.

E-mail address: kkholi@nagasaki-u.ac.jp (Kaio Kitazato).

Postal address: Division of Molecular Pharmacology of Infectious agents, Department of Molecular Microbiology and Immunology, Graduate School of Biomedical Sciences, Nagasaki University, 1-14 Bunkyo-machi, Nagasaki City, Nagasaki, 852-8521, Japan. 


\begin{abstract}
Influenza A virus (IAV) assembly and budding on host cell surface plasma membrane requires actin cytoskeleton reorganization. The underlying molecular mechanism involving actin reorganization remains unclarified. In this study, we found that the natural antiviral compound petagalloyl glucose (PGG) inhibits F-actin reorganization in the host cell membrane during the late stage of IAV infection, which are associated with the suppression of total cofilin-1 level and its phosphorylation. Knock-down of cofilin-1 reduces viral yields. These findings provide the first evidence that cofilin-1 plays an important role in regulating actin reorganization during IAV assembly and budding.
\end{abstract}

\title{
Highlights
}

- Influenza A virus (IAV) infection enhances F-actin aggregation on plasma membrane.

- Pentagalloyl glucose (PGG) inhibits F-actin aggregation and viral nucleoprotein accumulation on plasma membrane which was associated with suppressions of total cofilin-1 and its phosphorylation.

- Knock-down of cofilin-1 reduces IAV yields from host cells.

\section{Keywords}

Influenza A virus, viral assembly and budding, cofilin-1, F-actin, pentagalloyl glucose

\author{
Abbreviations \\ MOI, Multiplicity of infection; NP, nucleoprotein; HA, hemagglutinin; M1, matrix \\ protein 1; M2, matrix protein 2; vRNP, viral ribonucleoprotein; p.i., post infection; \\ PGG, pentagalloyl glucose
}




\section{Graphical Abstract (for review)}

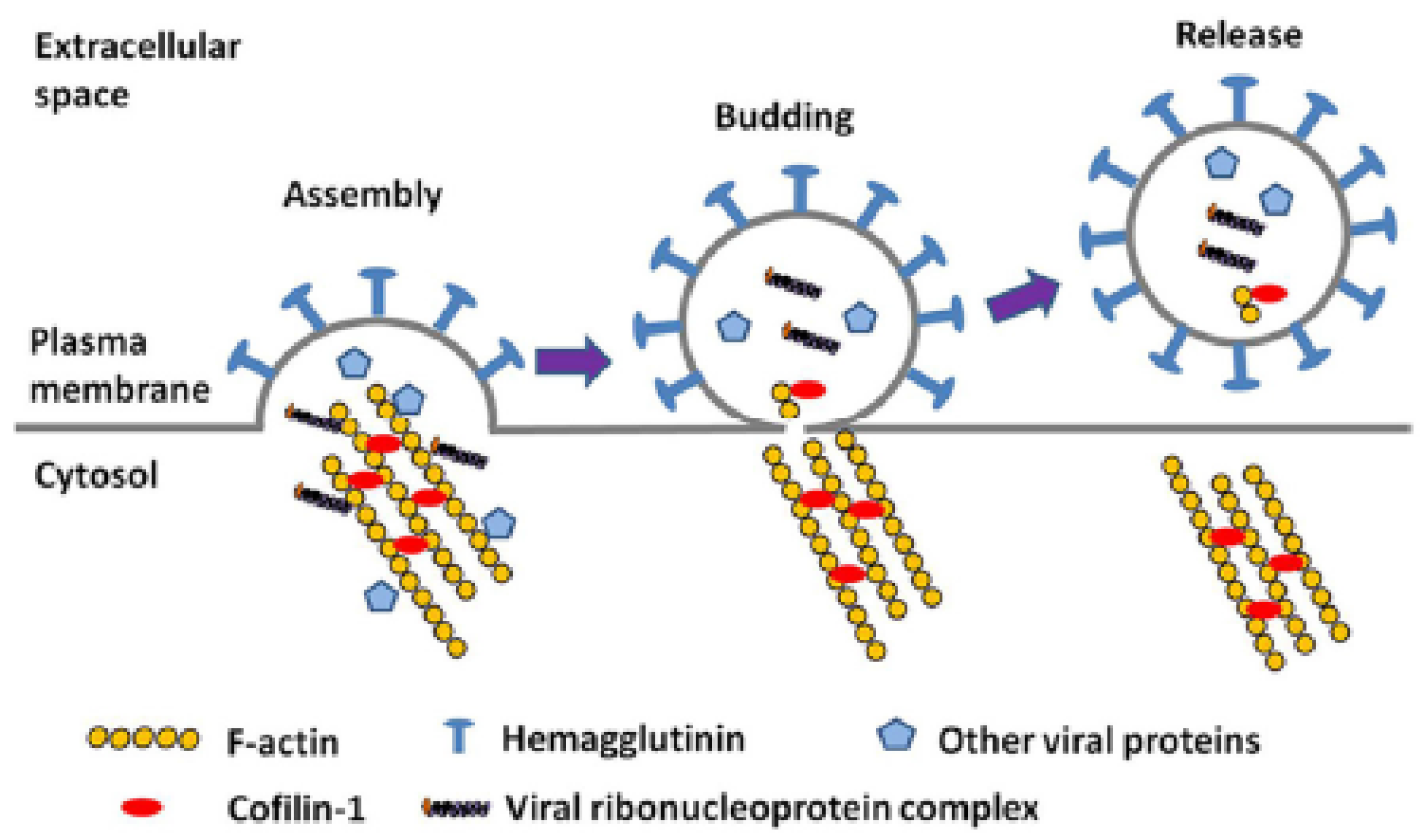




\section{Introduction}

Influenza A virus (IAV) remains a global health concern because of the emergence of novel virus subtypes or strains potentially capable of causing pandemics [1,2,3]. Due to high mutation rate during IAV replication, emergence of antiviral drug resistance is a major concern in influenza antiviral treatment [4], and highlights the need for new drug development. To avoid antiviral drug resistance, more attention is paid to find new targets for antiviral therapy that targeting host cell factors. This strategy requires understanding of molecular and cellular mechanisms involved in IAV infection and replication.

Host actin cytoskeleton has been reported to be involved in various stages of the IAV life cycle [5,6]. Actin filament depolymerization with CytoD and stabilization with jasplakinolide both inhibited influenza entry, suggesting that dynamic actin cytoskeletal reorganization is required for successful internalization of IAV into cells [5]. Actin cytoskeleton is also responsible for intracellular trafficking of IAV after entry, since treatment of cells with CytoD restricted intracellular viral movement [7]. Actin is associated with maintaining the lipid raft, where the assembly of IAV takes place, for incorporation into the budding filamentous virions [6]. Actin cytoskeleton interacts with several viral proteins (NP, M1 and M2) and provides the pushing force for incorporating the cytoplasmic vRNP complex into the budding site on plasma membrane $[8,9,10,11]$. A recent study revealed that influenza viral membrane protein HA clusters co-localize with actin-rich membrane regions and actin disruption caused specific changes to HA clustering [12]. Additionally, actin and actin-associated proteins are found in influenza virus particles [13]. These suggested that actin cytoskeleton also plays a critical role in the process of influenza virus assembly and budding.

Regulation of actin cytoskeleton requires several actin binding proteins, which organize the actin filaments (F-actin) into higher order structures such as actin bundles, branches, filopodia and microvilli [14]. The actin-depolymerizing factor (ADF)/cofilin family and gelsolin were previously reported to be essential regulators of actin cytoskeleton dynamics $[15,16]$. ADF/cofilin family consists of three highly similar paralogs: ADF (destrin), cofilin-1(non-muscle tissue) and cofilin-2 (muscle cofilin) [17]. ADF/cofilin family proteins accelerate the dissociation rate of actin subunits and server F-actin, which are primarily responsible for actin cytoskeleton remodeling [18]. The mammalian cofilin-1 is the most ubiquitous and involved in 
viral infection. HIV-1 inactivates cofilin to optimize immune evasion, replication, release, and cell-cell transmission [19,20,21]. We have previously reported that cofilin-1 play a crucial role in mediating biphasic F-actin dynamics to facilitate HSV1 infection and replication [22,23]. Cofilin-1 mediates the inhibition of actincytoskeleton remodeling and the anti-HSV activity of pentagalloyl glucose (PGG) [24]. Additionally, we found that PGG also inhibits IAV production by suppressing viral NP accumulation and virion budding on plasma membrane, which may be relevant to actin cytoskeleton remodeling [25]. Therefore, we speculated that cofilin-1 may play a role in IAV assembly and budding.

In this study, we found that IAV-induced F-actin aggregation on plasma membrane was possibly associated with total level of cofilin-1 protein and phosporylation, which were suppressed by PGG treatment. Moreover, we demonstrated that both cofilin-1 knock-down and PGG treatment can reduce viral yields. This study provides the first evidence that cofilin-1 is involved in regulation of actin reorganization during IAV assembly and budding.

\section{Materials and methods}

\subsection{Cells, virus and reagents}

MDCK and A549 cells were grown in DMEM or MEM (Invitrogen) supplemented with 10\% FBS (Cell Culture Bioscience) and antibiotics (100U/ml penicillin and $100 \mu \mathrm{g} / \mathrm{ml}$ streptomycin, Nacalai tesque). Influenza virus A/WSN/33 (H1N1) was propagated in 10-day-old embryonated chicken eggs. The allantoic fluids were harvested at 4 days after inoculation and stored at $-80^{\circ} \mathrm{C}$. Pentagalloyl glucose (PGG) was prepared as described previously [26].

\subsection{Laser scanning confocal immunofluorescence microscopy}

MDCK cells were infected with influenza A/WSN/33 (MOI=1) and then treated with DMSO or PGG. At $12 \mathrm{~h}$ p.i., cells were fixed with 4\% paraformaldehyde (Wako) and permeabilized with 1\%Triton X-100 (Nacalai tesque). F-actin was stained with TRITC-Phalloidin (50ng/ml, Sigma-Aldrich) for $15 \mathrm{~min}$. Viral NP protein was stained by using specific antibody against influenza A NP proteins (Santa Cruz Biotech). For deconvolution, a 63× Plan Apo objective was used for image acquisition and images were acquired at $0.5-\mu \mathrm{m}$ steps in the $\mathrm{z}$ axis using a laser scanning system, LSM710 (Carl Zeiss). 


\subsection{Transmission electron microscopy (TEM)}

MDCK cells were mock-infected or infected with influenza virus A/WSN/33(MOI=1). At 12h p.i., cells were fixed in 3\% glutaraldehyde ( $\mathrm{pH} 7.2$ ) for 1.5 hours, and post-fixed in 1\% osmium tetroxide for 1 hour. After dehydration, cells were embedded in Spurr's resin. Several consecutive ultrathin sections were cut on an LKB Nova ultramicrotome and then stained with saturated uranyl acetate and lead citrate. These sections were examined under a transmission electron microscope, JEM1400.

\subsection{Cofilin-1 siRNA preparation and transfection}

Cofilin-1 was knocked down with a specific targeting siRNA duplex consisting of oligo-DNAs with sequences 5'-GUCUUCAACGCCAGAGGAGTT-3' and 5'CUCCUCUGGCGUUGAAGACTT-3'. The scrambled siRNA duplex of oligo-DNAs 5'-UUCUCCGAACGUGUCACGUTT-3' and 5'-ACGUGACACGUUCGGAGAA TT-3' that does not target any gene product was used as a negative control. All siRNAs were obtained from Shanghai GenePharma Co., Ltd. A549 cells were transfected with $100 \mathrm{nM}$ siRNA in reduced serum medium OPTI-MEM-I (Invitrogen) according to the manufacturer's protocol. After 36 hours incubation, the cells were infected with influenza A/WSN/33 (MOI=1). Cells and culture supernatants were harvested at $12 \mathrm{~h}$ p.i. for Western blotting or virus titer quantification as previously reported [25].

\subsection{Western blotting}

Cell lysates were subjected to SDS-PAGE. Proteins were transferred to polyvinylidene fluoride (PVDF) membrane for Western blotting analysis, which was performed using specific antibodies against influenza A viral M1 proteins (Santa Cruz Biotech), $\beta$-actin(Sigma-Aldrich), $\alpha$-tubulin (Sigma-Aldrich), cofilin-1 (Calbiochem) or phosphorylated cofilin-1 (Sigma-Aldrich). Bound antibodies were visualized with an enhanced chemiluminescence (ECL) Plus Kit (GE Healthcare Life Sciences).

\subsection{Statistics analysis}

Statistical analysis was performed with Student's t-tests. Differences with P values of less than 0.05 were considered statistically significant. 


\section{Results}

3.1 IAV-induced aggregation of F-actin on cell surface membrane was suppressed by antiviral agent $P G G$

To confirm whether PGG can alter the surface actin filament-based structure, we performed a fluorescent TRITC phalloidin-labeled F-actin staining and TEM observation of infected cells in the presence or absence of PGG. As shown in Fig. 1A, mock-infected cells displayed sparse microvilli on the cell surface membrane, while more microvilli with expanded branches were observed in infected cells treated with or without DMSO (Fig. 1A upper panel), accompanied with aggregation of F-actin at margins of cells (Fig. 1A lower panel). In contrast, the IAV-induced microvilli-like protrusions and aggregation of F-actin was markedly inhibited by PGG treatment (Fig. 1A). The relative F-actin fluorescence intensity was analyzed semi-quantitatively and showed that PGG significantly inhibited IAV-induced augment of F-actin (Fig. 1B). We also examined the distributions of F-actin and viral NP protein on the surface plasma membrane of infected cells in the presence or absence of PGG using confocal laser scanning microscopy (Fig.1C). Z-axis reconstructions showed that increased accumulations of viral NP protein and co-localization with F-actin were found in the DMSO-treated cells, whereas the accumulations and co-localization were significantly reduced by PGG treatment (Fig.1D). Together, we concluded that IAVinduced microvilli-like structures on the surface of plasma membrane were associated with F-actin aggregation, which were significantly decreased by PGG treatment.

3.2 PGG did not alter expression level of actin but significantly reduced the expression level of cofilin-1 during IAV infection

We further confirmed the total expression levels of $\beta$-actin and cofilin-1 (regulator of actin cytoskeleton remodeling) in infected cells in the presence or absent of PGG by using Western blotting (Fig.2A). No significant changes in the total expression level of $\beta$-actin were observed either in mock-infected cells or IAVinfected cells in the presence of DMSO or PGG (in indicated concentrations) (Fig.2 B). However, the total expression level of cofilin-1 was significantly reduced by PGG treatment in a dose-dependent manner (Fig.2C). We calculated the ratio of cofilin-1 to actin was and found that the ratio was significantly increased after IAV infection but significantly suppressed by PGG treatment in a dose-dependent manner (Fig.2D). 


\subsection{PGG inhibits IAV-induced cofilin-1expression and phosphorylation}

We further examined the time-course effects of PGG treatment on total expression level and phosphorylation of cofilin-1 after IAV infection by using Western blotting (Fig.3A). Total cofilin-1 level was increased in IAV-infected cells treated with DMSO (Fig.3 B). In contrast, the total cofilin-1 level was timedependently reduced by PGG treatment (Fig.3B). Furthermore, IAV infection leads to cofilin-1 phosphorylation increase in DMSO-treated cells in a time-dependent manner, but the phosphorylated cofilin-1 (p-cofilin-1) level was markedly suppressed in PGGtreated infected cells (Fig.3C).

\subsection{Cofilin-1 is involved in IAV production}

To confirm whether cofilin-1 plays a role in IAV production, we examined the viral titer in culture supernatants from infected cells, which were treated by DMSO or PGG with different concentrations for 12h. As shown in Fig.4A, PGG dosedependently reduced virus yield. To further confirm the involvement of cofilin-1 in IAV production, we knocked down the endogenous cofilin-1 by siRNA, then infected the cells with influenza A/WSN/33 (MOI=1). As shown in Fig.4B, cofilin-1 siRNA significantly reduced their target mRNA level. Virus titer from the cofilin-knocked down cells was significantly reduced (Fig.4C), suggesting that cofilin is involved in virus production.

\section{Discussion}

Actin microfilaments (also called F-actin) are critical for the maturation step of many viruses [27,28,29]. Some host actin-binding proteins, such as Rab 11 and myosin, are essential for IAV genome transport to the plasma membrane and budding formation [30,31,32,33]. The major actin-depolymerizing factor (ADF)/cofilin isoform, Cofilin-1 is primarily responsible for dynamic actin cytoskeletal reorganization [15], and functions depending on its local concentration of active form relative to actin: relative low concentrations of active cofilin to actin favor severing, whereas high concentrations of cofilin favor nucleating actin assembly [34]. Cofilin-1 is deactivated by phosphorylation on Ser-3 by LIM-kinase1 (LIMK1), and results in an increase in F-actin [35, 36]. Disruption of the LIMK1-cofilin pathway affected viral releases of Mason-Pfizer monkey virus (M-PMV) and HIV-1 [21], as well as the 
entry and replication of HSV-1 [22,23,24]. However, there are no reports on IAV. In this study, we found that IAV infection enhanced surface cellular F-actin remodeling at the late stage of IAV replication cycle which was associated with an increase in the total expression and phosphorylation level of cofilin-1. We have previously shown that PGG treatment reduces cofilin-1 expression in HSV-infected cells [24], here we also found PGG can significantly reduce cofilin-1 expression in IAV-infected cells, suggesting cofilin-1 is involved in the antiviral effects of PGG against different viruses. The inhibitions of total and phosphorylation level of cofilin-1 by PGG may be associated with dynamic remodeling of F-actin in IAV-infected cells, subsequently leads to decrease in IAV production, which was also confirmed by cofilin-1 siRNA knockdown experiment.

IAV infection results in activation of phosphoinositide 3-kinase (PI3K) signaling pathway, which may inactivates cofilin and promote F-actin polymerization [23, 37, 38]. It has been reported that PGG reduces the expression of PI3K as well as phosphorylation of AKT and mammalian target of rapamycin (mTOR) [39]. It is likely that PGG suppress IAV-induced cofilin-1 phosphorylation via inhibition of the PI3K/Akt pathway.

In conclusion, our findings reveal that cofilin-1 plays an important role in mediating anti-IAV effects of PGG on viral assembly and budding during the late stage of IAV replication cycle. Understanding the underlying mechanism by which IAV regulates cofilin-1 activity to remodeling actin cytoskeleton may provide valuable insights into assembly and budding of IAV, leading to discovery of novel molecular target for anti-IAV drug development.

\section{Acknowledgements}

This work was supported by a Grant-in-aid for Scientific Research from the Japanese Society for the Promotion of Science, and a Grant-in-Aid for Scientific Research (C) from the Ministry of Education, Culture, Sports, Science and Technology of Japan (No.22590274 to K.K.), and partially supported by a Grant-inaid from the Tokyo Biochemical Research Foundation. 


\section{References}

[1] A. Gambotto, S.M. Barratt-Boyes, M.D. de Jong, G. Neumann, Y. Kawaoka, Human infection with highly pathogenic H5N1 influenza virus, Lancet 371 (2008) 1464-1475.

[2] N. Naffakh, S. van der Werf, April 2009: an outbreak of swine-origin influenza A(H1N1) virus with evidence for human-to-human transmission, Microbes Infect 11 (2009) 725-728.

[3] R. Gao, B. Cao, Y. Hu, Z. Feng, D. Wang, W. Hu, J. Chen, Z. Jie, H. Qiu, K. Xu, X. Xu, H. Lu, W. Zhu, Z. Gao, N. Xiang, Y. Shen, Z. He, Y. Gu, Z. Zhang, Y. Yang, X. Zhao, L. Zhou, X. Li, S. Zou, Y. Zhang, L. Yang, J. Guo, J. Dong, Q. Li, L. Dong, Y. Zhu, T. Bai, S. Wang, P. Hao, W. Yang, J. Han, H. Yu, D. Li, G.F. Gao, G. Wu, Y. Wang, Z. Yuan, Y. Shu, Human infection with a novel avian-origin influenza A (H7N9) virus, N Engl J Med 368 (2013) 1888-1897.

[4] J. Hsu, N. Santesso, R. Mustafa, J. Brozek, Y.L. Chen, J.P. Hopkins, A. Cheung, G. Hovhannisyan, L. Ivanova, S.A. Flottorp, I. Saeterdal, A.D. Wong, J. Tian, T.M. Uyeki, E.A. Akl, P. Alonso-Coello, F. Smaill, H.J. Schunemann, Antivirals for treatment of influenza: a systematic review and metaanalysis of observational studies, Ann Intern Med 156 (2012) 512-524.

[5] X. Sun, G.R. Whittaker, Role of the actin cytoskeleton during influenza virus internalization into polarized epithelial cells, Cell Microbiol 9 (2007) 1672-1682.

[6] M. Simpson-Holley, D. Ellis, D. Fisher, D. Elton, J. McCauley, P. Digard, A functional link between the actin cytoskeleton and lipid rafts during budding of filamentous influenza virions, Virology 301 (2002) 212-225.

[7] M. Lakadamyali, M.J. Rust, H.P. Babcock, X. Zhuang, Visualizing infection of individual influenza viruses, Proc Natl Acad Sci U S A 100 (2003) 9280-9285.

[8] R.T. Avalos, Z. Yu, D.P. Nayak, Association of influenza virus NP and M1 proteins with cellular cytoskeletal elements in influenza virus-infected cells, J Virol 71 (1997) 2947-2958.

[9] B. Thaa, A. Herrmann, M. Veit, Intrinsic cytoskeleton-dependent clustering of influenza virus M2 protein with hemagglutinin assessed by FLIM-FRET, J Virol 84 (2010) 12445-12449.

[10] P. Digard, D. Elton, K. Bishop, E. Medcalf, A. Weeds, B. Pope, Modulation of nuclear localization of the influenza virus nucleoprotein through interaction with actin filaments, J Virol 73 (1999) 22222231.

[11] D.P. Nayak, R.A. Balogun, H. Yamada, Z.H. Zhou, S. Barman, Influenza virus morphogenesis and budding, Virus Res 143 (2009) 147-161.

[12] M.V. Gudheti, N.M. Curthoys, T.J. Gould, D. Kim, M.S. Gunewardene, K.A. Gabor, J.A. Gosse, C.H. Kim, J. Zimmerberg, S.T. Hess, Actin mediates the nanoscale membrane organization of the clustered membrane protein influenza hemagglutinin, Biophys J 104 (2013) 2182-2192.

[13] M.L. Shaw, K.L. Stone, C.M. Colangelo, E.E. Gulcicek, P. Palese, Cellular proteins in influenza virus particles, PLoS Pathog 4 (2008) e1000085.

[14] S.J. Winder, K.R. Ayscough, Actin-binding proteins, J Cell Sci 118 (2005) 651-654.

[15] J.R. Bamburg, Proteins of the ADF/cofilin family: essential regulators of actin dynamics, Annu Rev Cell Dev Biol 15 (1999) 185-230.

[16] F.S. Southwick, Gelsolin and ADF/cofilin enhance the actin dynamics of motile cells, Proc Natl Acad Sci U S A 97 (2000) 6936-6938.

[17] B.W. Bernstein, J.R. Bamburg, ADF/cofilin: a functional node in cell biology, Trends Cell Biol 20 (2010) 187-195.

[18] T.D. Pollard, G.G. Borisy, Cellular motility driven by assembly and disassembly of actin filaments, Cell 112 (2003) 453-465.

[19] B. Stolp, M. Reichman-Fried, L. Abraham, X. Pan, S.I. Giese, S. Hannemann, P. Goulimari, E. Raz, R. Grosse, O.T. Fackler, HIV-1 Nef interferes with host cell motility by deregulation of Cofilin, Cell Host Microbe 6 (2009) 174-186.

[20] A. Yoder, D. Yu, L. Dong, S.R. Iyer, X. Xu, J. Kelly, J. Liu, W. Wang, P.J. Vorster, L. Agulto, D.A. Stephany, J.N. Cooper, J.W. Marsh, Y. Wu, HIV envelope-CXCR4 signaling activates cofilin to overcome cortical actin restriction in resting CD4 T cells, Cell 134 (2008) 782-792.

[21] X. Wen, L. Ding, J.J. Wang, M. Qi, J. Hammonds, H. Chu, X. Chen, E. Hunter, P. Spearman, ROCK1 and LIM Kinase Modulate Retrovirus Particle Release and Cell-Cell Transmission Events, J Virol 88 (2014) 6906-6921.

[22] Y. Xiang, K. Zheng, H. Ju, S. Wang, Y. Pei, W. Ding, Z. Chen, Q. Wang, X. Qiu, M. Zhong, F. Zeng, Z. Ren, C. Qian, G. Liu, K. Kitazato, Y. Wang, Cofilin 1-mediated biphasic F-actin dynamics of neuronal 
cells affect herpes simplex virus 1 infection and replication, J Virol 86 (2012) 8440-8451.

[23] K. Zheng, Y. Xiang, X. Wang, Q. Wang, M. Zhong, S. Wang, X. Wang, J. Fan, K. Kitazato, Y. Wang, Epidermal growth factor receptor-PI3K signaling controls cofilin activity to facilitate herpes simplex virus 1 entry into neuronal cells, MBio 5 (2014) e00958-00913.

[24] Y. Pei, Y.F. Xiang, J.N. Chen, C.H. Lu, J. Hao, Q. Du, C.C. Lai, C. Qu, S. Li, H.Q. Ju, Z. Ren, Q.Y. Liu, S. Xiong, C.W. Qian, F.L. Zeng, P.Z. Zhang, C.R. Yang, Y.J. Zhang, J. Xu, K. Kitazato, Y.F. Wang, Pentagalloylglucose downregulates cofilin1 and inhibits HSV-1 infection, Antiviral Res 89 (2011) 98-108.

[25] G. Liu, S. Xiong, Y.F. Xiang, C.W. Guo, F. Ge, C.R. Yang, Y.J. Zhang, Y.F. Wang, K. Kitazato, Antiviral activity and possible mechanisms of action of pentagalloylglucose (PGG) against influenza A virus, Arch Virol 156 (2011) 1359-1369.

[26] Y.-J. Zhang, T. NAGAO, T. TANAKA, C.-R. YANG, H. OKABE, I. KOUNO, Antiproliferative Activity of the Main Constituents from Phyllanthus emblica, Biol. Pharm. Bull 27(2) (2004) 251-255.

[27] J.J. Chu, B.G. Choo, J.W. Lee, M.L. Ng, Actin filaments participate in West Nile (Sarafend) virus maturation process, J Med Virol 71 (2003) 463-472.

[28] S.M. Eswarappa, V. Pareek, D. Chakravortty, Role of actin cytoskeleton in LPS-induced NF-kappaB activation and nitric oxide production in murine macrophages, Innate Immun 14 (2008) 309-318.

[29] C.N. Gujuluva, A. Kundu, K.G. Murti, D.P. Nayak, Abortive replication of influenza virus A/WSN/33 in HeLa229 cells: defective viral entry and budding processes, Virology 204 (1994) 491-505.

[30] E.A. Bruce, P. Digard, A.D. Stuart, The Rab11 pathway is required for influenza a virus budding and filament formation, J Virol 84 (2010) 5848-5859.

[31] M.J. Amorim, E.A. Bruce, E.K. Read, A. Foeglein, R. Mahen, A.D. Stuart, P. Digard, A rab11- and microtubule-dependent mechanism for cytoplasmic transport of influenza a virus viral RNA, J Virol 85 (2011) 4143-4156.

[32] A.J. Eisfeld, E. Kawakami, T. Watanabe, G. Neumann, Y. Kawaoka, RAB11A is Essential for Influenza Genome Transport to the Plasma Membrane, J Virol (2011).

[33] M. Haidari, W. Zhang, L. Ganjehei, M. Ali, Z. Chen, Inhibition of MLC phosphorylation restricts replication of influenza virus--a mechanism of action for anti-influenza agents, PLoS One 6 (2011) e21444.

[34] E. Andrianantoandro, T.D. Pollard, Mechanism of actin filament turnover by severing and nucleation at different concentrations of ADF/cofilin, Mol Cell 24 (2006) 13-23.

[35] S. Arber, F.A. Barbayannis, H. Hanser, C. Schneider, C.A. Stanyon, O. Bernard, P. Caroni, Regulation of actin dynamics through phosphorylation of cofilin by LIM-kinase, Nature 393 (1998) 805-809.

[36] N. Yang, O. Higuchi, K. Ohashi, K. Nagata, A. Wada, K. Kangawa, E. Nishida, K. Mizuno, Cofilin phosphorylation by LIM-kinase 1 and its role in Rac-mediated actin reorganization, Nature 393 (1998) 809-812.

[37] C. Ehrhardt, H. Marjuki, T. Wolff, B. Nurnberg, O. Planz, S. Pleschka, S. Ludwig, Bivalent role of the phosphatidylinositol-3-kinase (PI3K) during influenza virus infection and host cell defence, Cell Microbiol 8 (2006) 1336-1348.

[38] G. Nebl, S. Fischer, R. Penzel, Y. Samstag, Dephosphorylation of cofilin is regulated through Ras and requires the combined activities of the Ras-effectors MEK and PI3K, Cell Signal 16 (2004) 235243.

[39] K.Y. Park, H.J. Lee, S.J. Jeong, H.S. Kim, S.H. Kim, S. Lim, H.C. Kim, J. Lu, 1,2,3,4,6-Penta-O-gallolybeta-D-glucose suppresses hypoxia-induced accumulation of hypoxia-inducible factor-1alpha and signaling in LNCaP prostate cancer cells, Biol Pharm Bull 33 (2010) 1835-1840. 


\section{Figure legends}

Fig.1 IAV-induced aggregation of F-actin on cell surface was suppressed by antiviral agent PGG. (A) MDCK cells were mock-infected or infected with influenza A/WSN/33 (MOI=1) in the presence or absence of PGG $(12.5 \mu \mathrm{g} / \mathrm{ml})$ or DMSO $(0.1 \%, v / v)$. At $12 \mathrm{~h}$ p.i., cells were examined with a transmission electron microscopy (scale bar $=1 \mu \mathrm{m}$ ). After stainning with Phalloidin-TRITC, the pattern of F-actin in cells was imaged using a confocal laser scanning microscopy. (B) Semiquantification of F-actin accumulated on plasma membrane in infected cells in the presence or absence of PGG. (C) F-actin (stained with Phalloidin-TRITC in red) and viral NP protein (stained with anti-viral NP antibodies in green) was imaged by confocal laser scanning microscopy. Representative z-plane reconstructions (xz-axis) are shown (Scale bar $=20 \mu \mathrm{m})$. Areas of colocalization are evidenced in yellow. (D) Semi-quantification of viral NP protein accumulated on plasma membrane in infected cells in the presence or absence of PGG. Each value represents the mean and SD of three separate experiments $(* \mathrm{P}<0.05)$.

Fig.2 PGG did not alter expression level of actin but significantly reduced expression level of cofilin-1 during IAV infection. (A) A549 cells were infected with A/WSN/33 (MOI=1) and subsequently treated with PGG (indicated concentration) or DMSO $(0.1 \%$, v/v). At $12 \mathrm{~h}$ p.i., cells were analyzed by Western blotting analysis to determine the expressions of viral M1, cofilin-1, $\beta$-actin and $\alpha$ tubulin proteins. $(\mathrm{B}, \mathrm{C})$ The relative expression levels of $\beta$-actin and cofilin-1 normalizing to that of $\alpha$-tubulin. (D) The ratio of total cofilin- 1 to $\beta$-actin was calculated and represented. Each value represents the mean and SD of three separate experiments $(* \mathrm{P}<0.05)$.

Fig.3 PGG inhibits IAV-induced cofilin-1 expression and phosphorylation. (A) A549 cells were infected with A/WSN/33 (MOI=1) and subsequently treated with PGG $(12.5 \mu \mathrm{g} / \mathrm{ml})$ or DMSO $(0.1 \%, \mathrm{v} / \mathrm{v})$. At $0,3,6,9,12$ and $24 \mathrm{~h}$ p.i., cells were lysed and subjected to Western blotting to determine the expression of viral M1, cofilin-1, phorsphorylated cofilin-1 and $\alpha$-tubulin proteins. Relative expression level of cofilin-1 (B) and phosphorylated cofilin-1 (C) was calculated by normalizing to that of $\alpha$-tubulin, respectively. Each value represents the mean and SD of three 
separate experiments $(* \mathrm{P}<0.05)$.

Fig.4 Cofilin-1 is involved in influenza virus production. (A) A549 cells were infected with A/WSN/33 (MOI=1) and subsequently treated with PGG (indicated concentrations) or DMSO $(0.1 \%$, v/v). At 12 h p.i., culture supernatant was harvested and the virus titer was determined by plaque assay. (B) A549 cells were transfected with siRNAs for 36h, and then infected with influenza A/WSN/33(MOI=1) for $12 \mathrm{~h}$ p.i.. Expression of cofilin-1 was determined by Western blotting. si.NC and si.cofilin present scrambled siRNA and cofilin-1 siRNA respectively. (C) Viral titers from different culture supernatants were determined by plaque assay. Each value represents the mean and SD of three separate experiments $(* \mathrm{P}<0.05)$. 
Figure 1

A

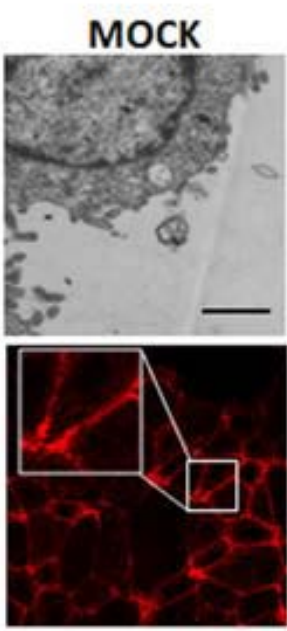

$\overline{\mathrm{DMSO}-\mathrm{PGG} \text { - DMSO+ PGG - DMSO+ PGG }}+$

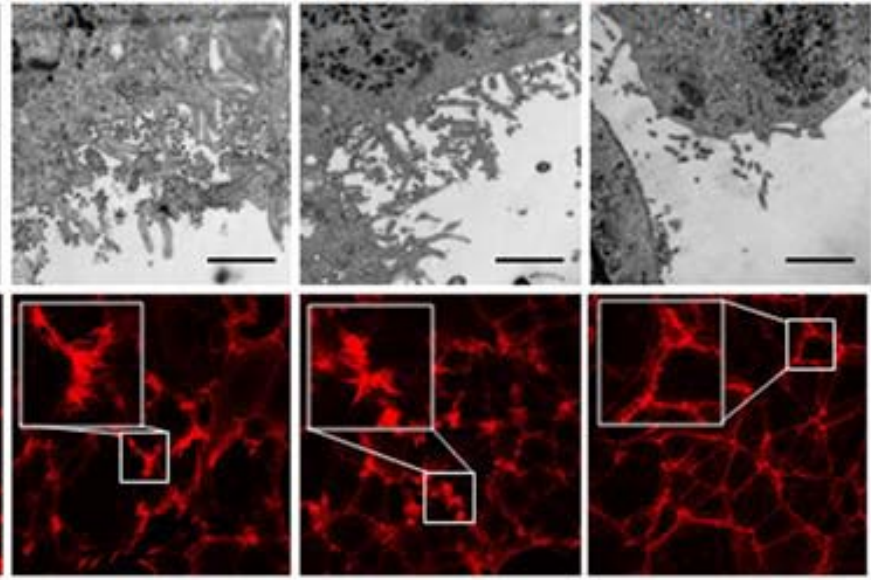

C

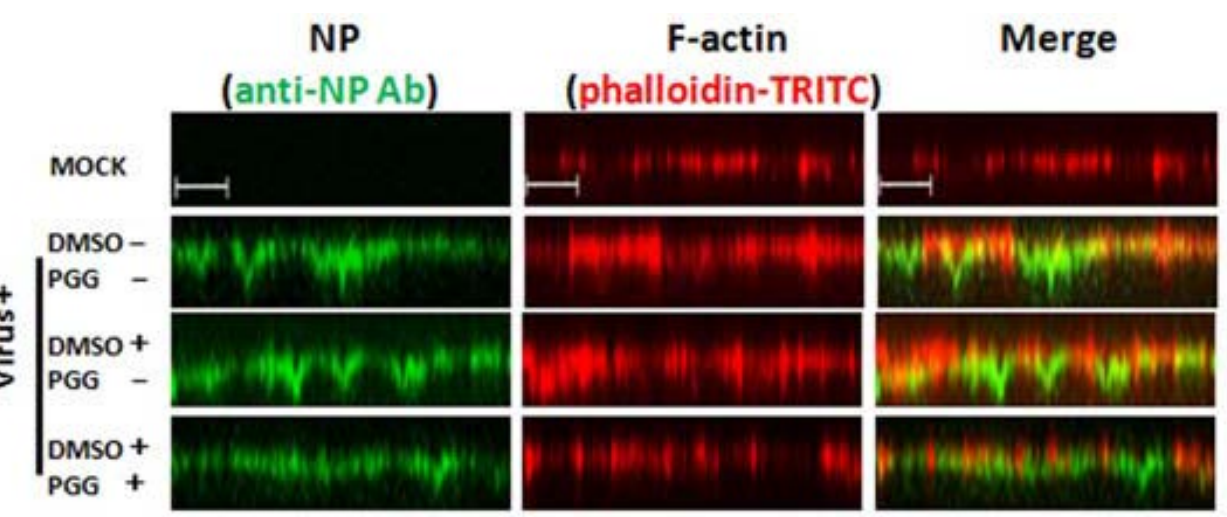

B

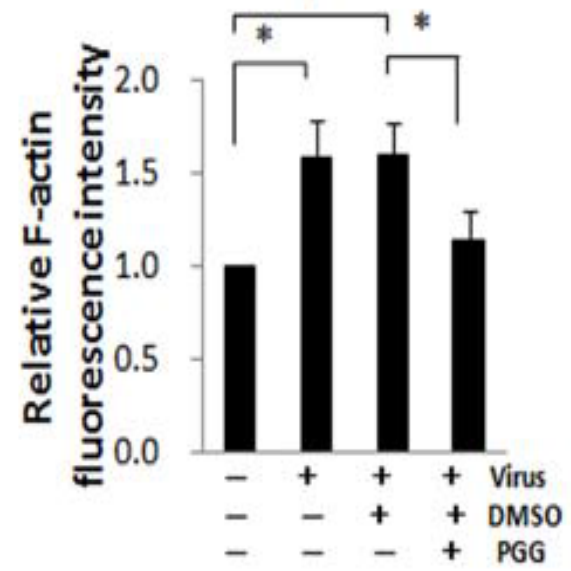

D

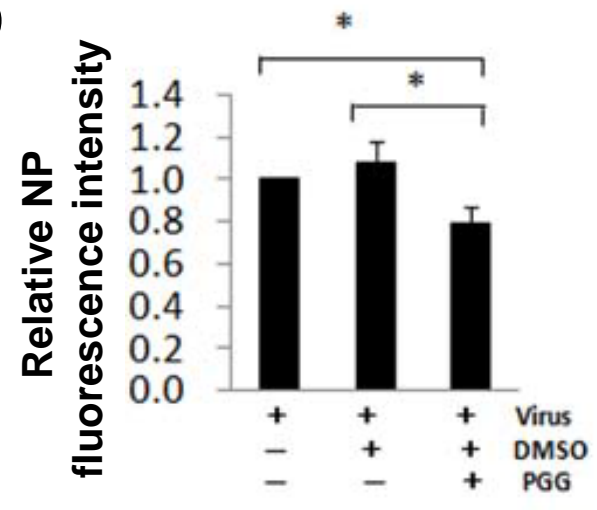




\section{Figure 2}

A

A/WSN/33 infection

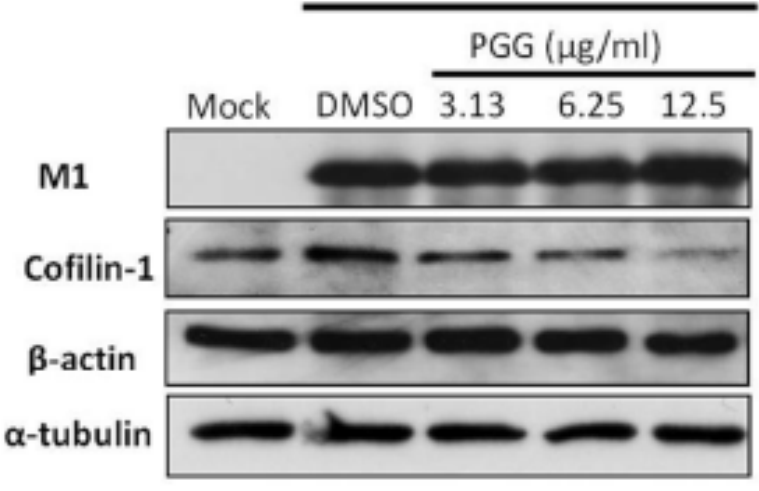

C

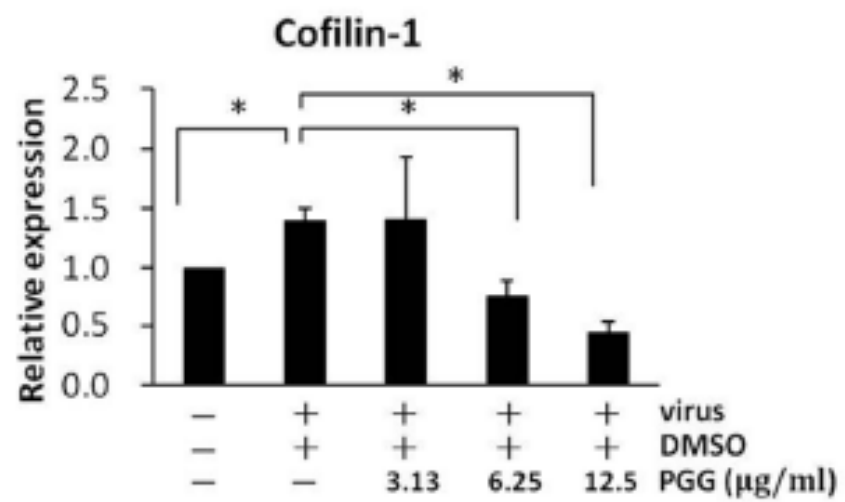

B

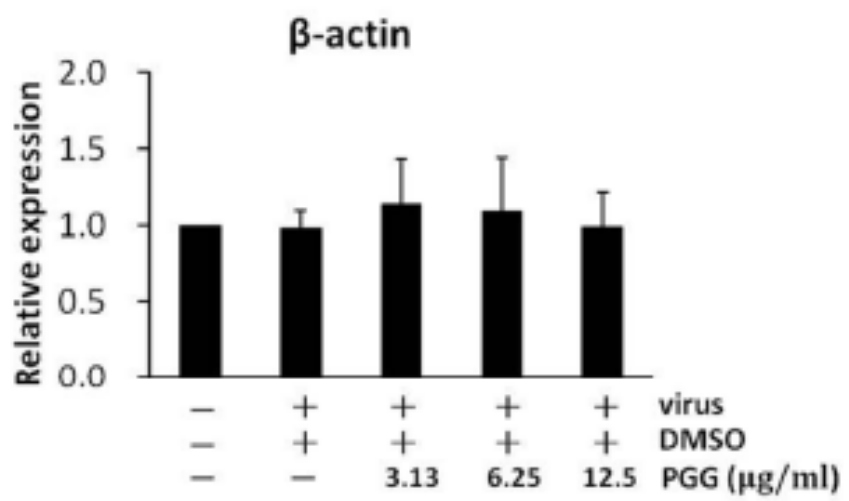

D

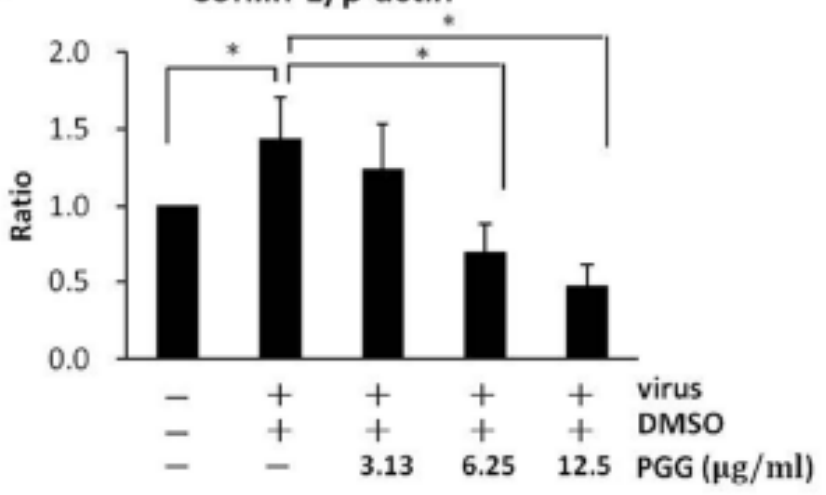




\section{Figure 3}

A

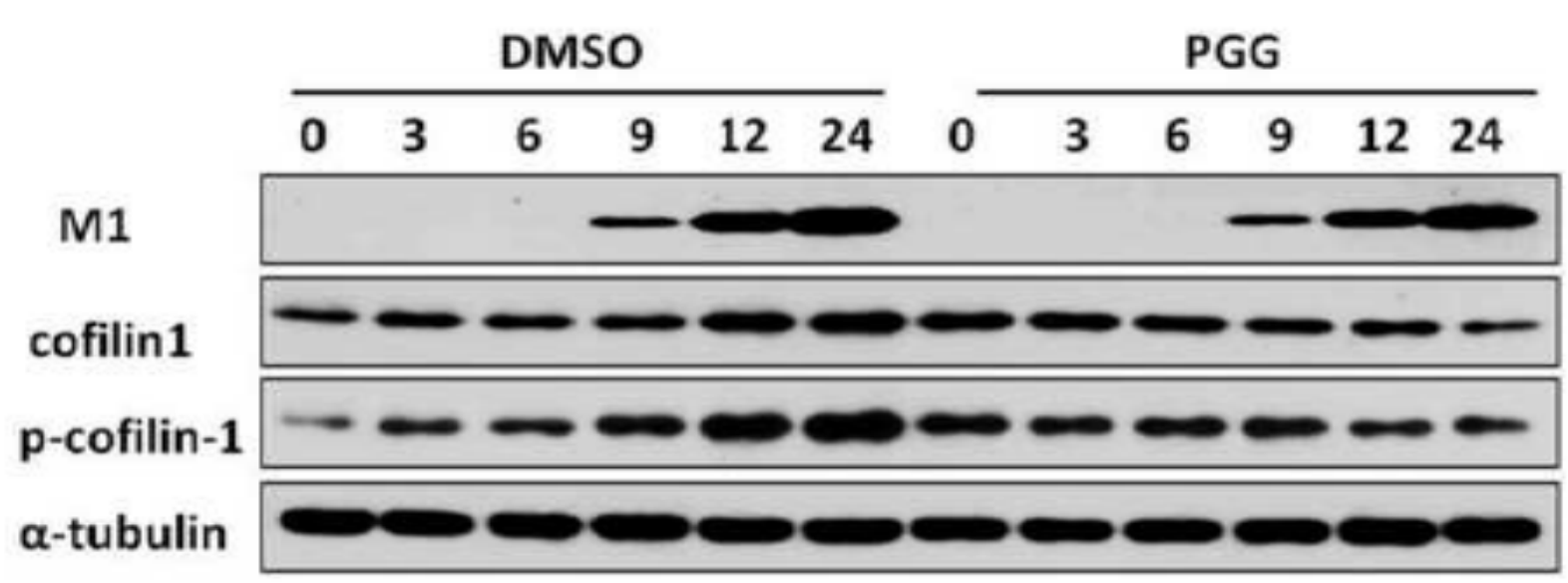

B

cofilin-1

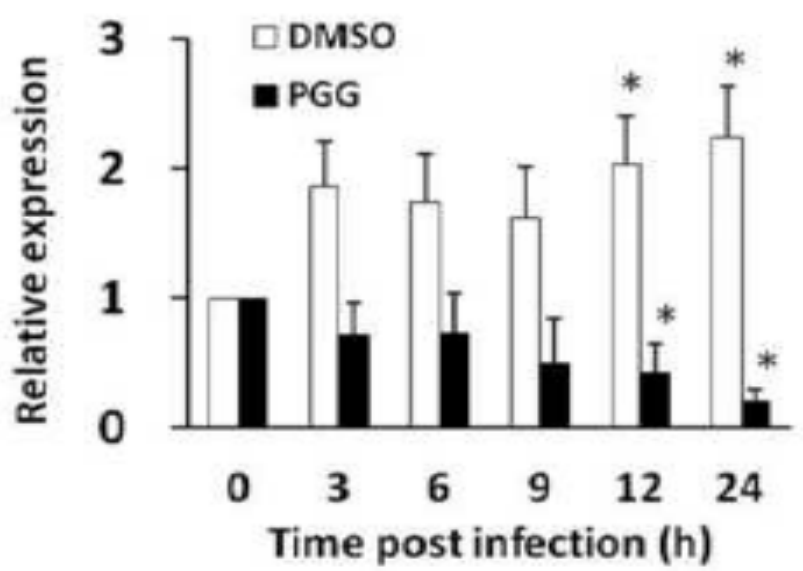

C

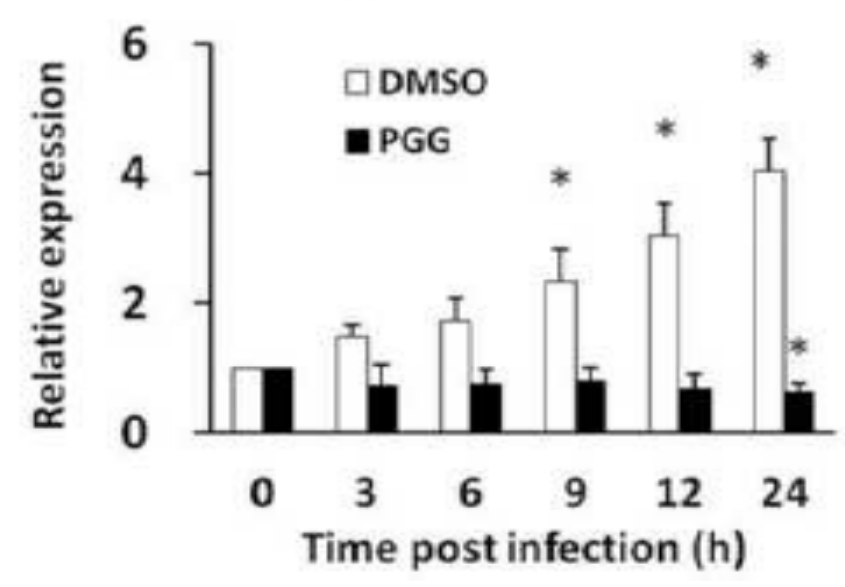


Figure 4
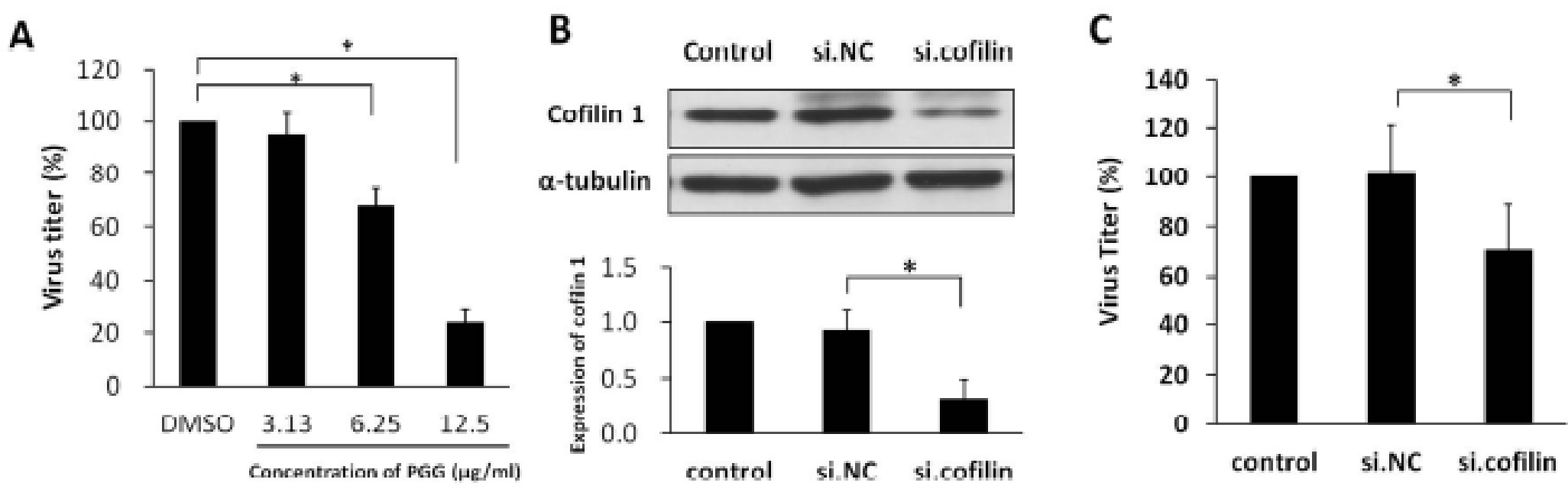